\title{
Multimorbidity, a challenge for medical treatment, research and health care management
}

\author{
Type of article: Conference abstract
}

\author{
Wolfgang Seger \\ Chair of the Health Sciences Section of the Medical Technologies Journal, \\ Germany.
}

\begin{abstract}
Multimorbidity is a highly prevalent phenomenon in the elderly and of growing public health impact in aging societies due to rising life expectancies, more effective medical treatment and comprehensive social care facilities, resulting in longevity[1-3].

The increasing number of patients at an age of 60 and more are expected to have between 5 to 7 active diagnoses with multiple conditions and pathological states, varying recommendations for therapy and diverse personal wishes and contextual factors influencing. Disease-disease interactions have an impact on patient management processes, disease-disease medication interactions must be considered and balanced and potential medication-medication interactions kept in mind and avoided. Furthermore the interaction between somatic and psychiatric diseases is a completely underestimated field of conflicts and misjudgements. Multimorbidity is an important field of research as well. Thusthe lack of guidelines covering multimorbidity is more the rule than an exemption if not explicitely addressing old age.

Multimorbidity is going along with multiple changing of medical consultations, the need for a comprehensive care by different professions with time-consuming and strenuous transitions and communication routes, repeated hospital treatments, polypharmacy with frequently undesired mutual actions of drugs, unnecessary or redundant examinations and conflicting instructions for therapy resulting in accumulating complications unless rationally analyzed.

Multimorbidity is more than an addition of different diseases. It is a complex net of mutually influencing factors like physical functioning, mental well-being, social relationships and environmental factors. Thereforetherapy of multimorbidity is more sensitive for contextual factors, needs multimodal and multidisciplinary support with attention for the patients' resources and an increasing need for prevention, therapy, rehabilitation, assistive technologies and social care at the place of residence or nearby [4].
\end{abstract}

Key words: Multimorbidity, Public Health, Aging, Life Expectancy, Contextual Factors.

\section{Conflict of intereststatement}

This article is a keynote presented at the International Congress on Health Sciences and Medical Technologies 2018 ICHSMT'18. 


\section{Authors' biography}

Professor Dr. med. Wolfgang Seger is a specialist in Internal Medicine, Gastroenterology, Rehabilitation and Social Medicine. He is Chairman of the Health Advisory Board of the Federal Rehabilitation Council in Germany. He recently retired from the Directory of the Health and Long-Term-Care Advisory Board of all Social Health Insurances in Lower Saxony, Germany, which he served for more than 25 years. Prof. Seger is chair of the Health Sciences Section of the Medical Technologies Journal.

\section{References}

[1]National Institute for Health and Care Excellence (2016). Multimorbidity: clinical assessment and management. NG 56. https://www.nice.org.uk/guidance/ng56

[2]The Academy of Medical Sciences (2018). Multimorbidity: a priority for global health research.

http://acmedsci.ac.uk/policy/policy-projects/multimorbidity

[3]World Health Organisation (2016). Multimorbidity: technical series on safer primary care.

http://www.who.int/patientsafety/topics/primary-care/technical_series/en/

[4]Seger W (2018).Future trends in medical rehabilitation in the context of multimorbidity - Part I: definition of terms, issues and challenges.

Gesundheitswesen 80;12-19, Part II: structuralrequirementstomanage an

appropriaterehabilitative healthcare. Gesundheitswesen 80;105-112 